\title{
(GIGA)bYte
}

\section{In silico characterization of chitin deacetylase genes in the Diaphorina citri genome}

\author{
Sherry Miller ${ }^{1,2}$, Teresa D. Shippy ${ }^{1}$, Blessy Tamayo ${ }^{3}$, Prashant S. Hosmani ${ }^{4}$, \\ Mirella Flores-Gonzalez ${ }^{4}$, Lukas A. Mueller ${ }^{4}$, Wayne B. Hunter ${ }^{5}$, \\ Susan J. Brown ${ }^{1}$, Tom D’Elia ${ }^{3}$ and Surya Saha ${ }^{4,6, *}$ \\ 1 Division of Biology, Kansas State University, Manhattan, KS 66506, USA \\ 2 Allen County Community College, Burlingame, KS 66413, USA \\ 3 Indian River State College, Fort Pierce, FL 34981, USA \\ 4 Boyce Thompson Institute, Ithaca, NY 14853, USA \\ 5 USDA-ARS, U.S. Horticultural Research Laboratory, Fort Pierce, FL 34945, USA \\ 6 Animal and Comparative Biomedical Sciences, University of Arizona, Tucson, AZ 85721, USA
}

\section{ABSTRACT}

Chitin deacetylases (CDAs) are one of the least understood components of insect chitin metabolism. The partial deacetylation of chitin polymers appears to be important for the proper formation of higher order chitin structures, such as long fibers and bundles, which contribute to the integrity of the insect exoskeleton and other structures. Some CDAs may also be involved in bacterial defense. Here, we report the manual annotation of four CDA genes from the Asian citrus psyllid, Diaphorina citri, laying the groundwork for future study of these genes.

Subjects Genetics and Genomics, Animal Genetics, Bioinformatics

Submitted: 23 December 2020 Accepted: $\quad 09$ June 2021 Published: 11 June 2021

* Corresponding author. E-mail: ss2489@cornell.edu

Published by GigaScience Press.

Preprint submitted at https: //doi.org/10.1101/2020.12.22.424074

This is an Open Access article distributed under the terms of the Creative Commons Attribution License (http://creativecommons.org/ licenses/by/4.0/), which permits unrestricted reuse, distribution, and reproduction in any medium, provided the original work is properly cited.

Gigabyte, 2021, 1-11

\section{DATA DESCRIPTION}

\section{Introduction}

Chitin deacetylases (CDAs) are metalloenzymes that partially deacetylate chitin polymers [1]. CDA activity in insects was first reported in the cabbage looper Trichoplusia ni [2]. In Drosophila melanogaster, several CDAs are involved in tracheal development [3, 4]. More recently, genomic and phylogenetic studies have shown that CDAs are present widely in insects and can be classified into five different groups [5, 6]. Most holometabolous insects have at least one representative of each of the five CDA groups, while the hemimetabolous insects that have been examined lack group II and group V genes [6]. The exact role of insect CDAs is not well understood, but they may play a role in organization of chitin molecules into higher order structures [7].

\section{Context}

Loss of function experiments indicate that some CDAs are essential for growth and development, making them a potential target for insect pest control [6, 8-12]. Here we describe the chitin deacetylase gene family in the Asian citrus psyllid, Diaphorina citri (Hemiptera: Liviidae; NCBI:txid121845). D. citri is the vector for Candidatus Liberibacter asiaticus (CLas), which is responsible for the global outbreak of Huanglongbing (citrus greening) disease. We identified four chitin deacetylase genes in the D. citri v3 genome, 


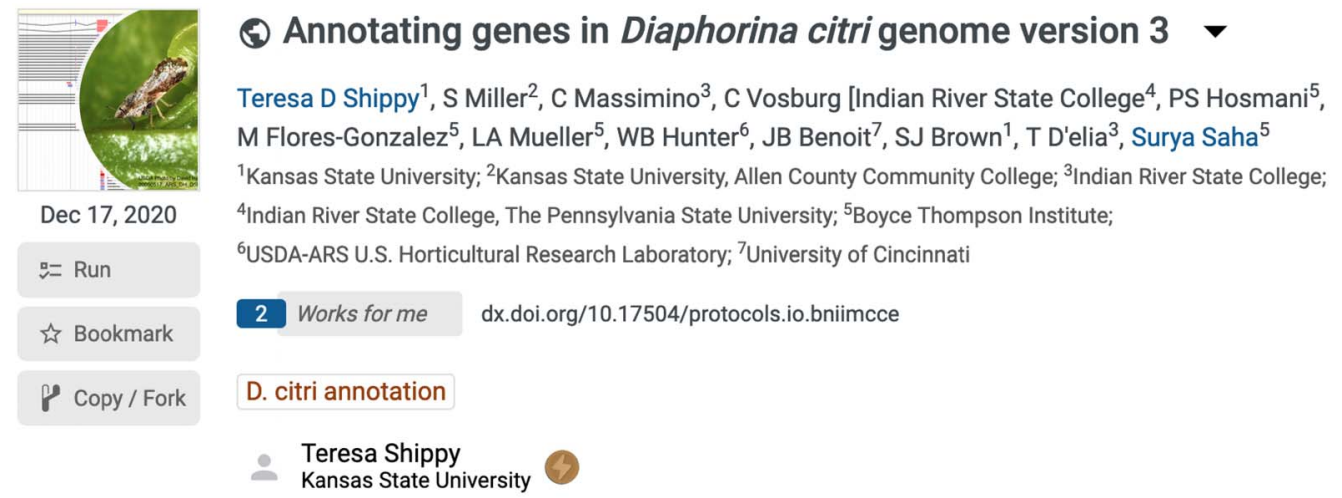

Figure 1. Protocols.io protocol outlining the annotation process of the $D$. citri genome [16]. https://www.protocols. io/widgets/doi?uri=dx.doi.org/10.17504/protocols.io.bniimcce

three of which have multiple isoforms. As in other hemipterans, only groups I, III and IV are represented [6].

\section{METHODS}

Chitin deacetylase genes in D. citri genome v3 [13] were identified by BLAST (NCBI BLAST, RRID:SCR_004870) search of $D$. citri sequences with chitin deacetylase orthologs from other insects. Orthology was confirmed by reciprocal BLAST of the National Center for Biotechnology Information (NCBI) non-redundant protein database [14]. Genes were manually annotated in Apollo 2.1.0 (RRID:SCR_001936) [15] using available evidence, including RNA-seq reads, Iso-seq transcripts and de novo-assembled transcripts. A more detailed annotation protocol is available at protocols.io (Figure 1) [16].

Domain analysis was performed with InterPro (InterPro, RRID:SCR_006695) [17]. Multiple alignments were performed using CLUSTALW (RRID:SCR_002909) [18] within MEGA X (MEGA software, RRID:SCR_000667) [19] followed by phylogenetic tree construction using the neighbor-joining method in MEGA X. Table 1 contains a list of orthologs used in the phylogenetic analysis. Expression data from the Citrus Greening Expression Network (CGEN) [20] (Table 2) was visualized using the pheatmap (pheatmap, RRID:SCR_016418) package of R (R Project for Statistical Computing, RRID:SCR_001905) [21, 22] or Microsoft Excel (Microsoft Excel, RRID:SCR_016137).

\section{DATA VALIDATION AND QUALITY CONTROL}

Chitin deacetylase genes in the $D$. citri v3 genome [13] were identified and manually annotated as described below. These genes were classified following the precedents established in other insects [5,6].

\section{Group I chitin deacetylases}

Most insects have two group I genes named $C D A 1$ and $C D A 2$ (Table 3). The proteins encoded by these genes have an $\mathrm{N}$-terminal chitin-binding domain (ChBD), a low-density lipoprotein receptor class A domain (LDLa), and a deacetylase catalytic domain [5]. RNA interference (RNAi) of group I CDAs in a variety of insects suggests that loss of function of CDA1 or CDA2 can result in lethality and therefore these genes could be potential targets for pest control 


\begin{tabular}{|c|c|c|c|}
\hline Species & Accession & Name in NCBI & Name in Tree \\
\hline Nilaparvata lugens & AJQ20732.1 & chitin deacetylase 1 & Nl CDA1 \\
\hline Nilaparvata lugens & AJQ20733.1 & chitin deacetylase 2 & $\mathrm{Nl}$ CDA2 \\
\hline Nilaparvata lugens & AJQ20734.1 & chitin deacetylase 3 & $\mathrm{Nl}$ CDA3 \\
\hline Nilaparvata lugens & AJQ20735.1 & chitin deacetylase 4 & $\mathrm{Nl}$ CDA4 \\
\hline Tribolium castaneum & NP_001095946.1 & chitin deacetylase 1 precursor & Tc CDA1 \\
\hline Tribolium castaneum & NP_001116303.1 & chitin deacetylase 2 isoform $\mathrm{B}$ precursor & Tc CDA2 \\
\hline Tribolium castaneum & NP_001104011.1 & chitin deacetylase 3 precursor & Tc CDA3 \\
\hline Tribolium castaneum & NP_001103903.1 & chitin deacetylase 4 precursor & Tc CDA4 \\
\hline Tribolium castaneum & NP_001103739.1 & chitin deacetylase 5 isoform A precursor & Tc CDA5 \\
\hline Tribolium castaneum & NP_001103905.1 & chitin deacetylase 6 precursor & Tc CDA6 \\
\hline Tribolium castaneum & NP_001104012.1 & chitin deacetylase 7 precursor & Tc CDA7 \\
\hline Tribolium castaneum & NP_001103906.1 & chitin deacetylase 8 precursor & Tc CDA8 \\
\hline Tribolium castaneum & NP_001103904.1 & chitin deacetylase 9 precursor & Tc CDA9 \\
\hline Drosophila melanogaster & NP_001262062.1 & serpentine, isoform C & Dm Serp \\
\hline Drosophila melanogaster & NP_730442.2 & vermiform, isoform G & Dm verm \\
\hline Drosophila melanogaster & NP_609806.1 & ChLD3 & Dm ChLD3 \\
\hline Drosophila melanogaster & NP_728468.1 & chitin deacetylase-like 4 & Dm Cda4 \\
\hline Drosophila melanogaster & NP_001245808.1 & chitin deacetylase-like 5 , isoform I & Dm Cda5 \\
\hline Drosophila melanogaster & NP_001286519.1 & chitin deacetylase-like 9 , isoform $B$ & Dm Cda9 \\
\hline
\end{tabular}

methods [6-12]. Recent experiments in Tribolium suggest that TcCDA1 and TcCDA2 are required for organization of chitin into longer fibers that are important for cuticular strength [7].

As expected, we identified two group I genes in $D$. citri, which we named $C D A 1$ and $C D A 2$. Both genes encode proteins with the typical group I domain structure (Figure 3). We identified two isoforms each for D. citri $C D A 1$ and $C D A 2$ (Table 4). $C D A 2$ has previously been shown to have multiple isoforms in several holometabolous insect species, with the transcripts differing only in the use of one alternative exon [5, 10,12]. This gene structure is conserved in $D$. citri $C D A 2$ with alternate exons $3 \mathrm{a}$ and $3 \mathrm{~b}$. The two $D$. citri $C D A 1$ isoforms differ in the presence or absence of a 24-bp exon upstream of the last exon. Expression data from RNA-seq datasets available through CGEN [20] suggest that, in general, expression of CDA1 and CDA2 is higher in nymphs and eggs than in adults (Figure 2A).

In Drosophila and Tribolium, the CDA1 and CDA2 orthologs are adjacent to one another in the genome [3,5] on chromosomes 3 and 5, respectively. The conserved clustering of these genes suggests there may be evolutionary constraint on their physical location. We found that the $D$. citri $C D A 1$ and $C D A 2$ orthologs are also adjacent to one another on chromosome 4 . In the $D$. citri v3 genome, these genes are separated by approximately 50 kilobase pairs $(\mathrm{Kb})$, although this distance appears to be inflated by falsely duplicated fragments of both genes in this assembly.

\section{Group III chitin deacetylases}

We identified one group III CDA in the $D$. citri v3 genome (Tables 3 and 4). This gene has been previously described and was named $C D A 3$ because of its orthology to Nilaparvata lugens CDA3 [33]. Like group III CDAs in other insects, D. citri CDA3 contains a ChBD and catalytic domain but lacks the low-density lipoprotein receptor class A (LDLa) domain found in group I CDAs (Figure 3). Improvements in the genome assembly mean that our 


\begin{tabular}{|c|c|c|c|c|c|c|c|c|c|c|}
\hline Gene ID & $\begin{array}{l}\text { Dcitr04g03 } \\
\text { 590.1.1 }\end{array}$ & $\begin{array}{l}\text { Dcitr04g03 } \\
590.1 .2\end{array}$ & $\begin{array}{l}\text { Dcitr04g03 } \\
\text { 540.1.1 }\end{array}$ & $\begin{array}{l}\text { Dcitr04g03 } \\
540.1 .2\end{array}$ & $\begin{array}{l}\text { Dcitr02g03 } \\
950.1 .1\end{array}$ & $\begin{array}{l}\text { Dcitr01g12 } \\
\text { 310.1.1 }\end{array}$ & $\begin{array}{l}\text { Dcitr01g12 } \\
\text { 310.1.2 }\end{array}$ & $\begin{array}{l}\text { Dcitr01g12 } \\
\text { 310.1.3 }\end{array}$ & $\begin{array}{l}\text { Dcitr01g12 } \\
\text { 310.1.4 }\end{array}$ & $\begin{array}{l}\text { Dcitr01g12 } \\
\text { 310.1.5 }\end{array}$ \\
\hline Gene/Transcript Name & CDA1-RB & CDA1-RA & CDA2-RB & CDA2-RA & CDA4 & CDA5-RD & $\begin{array}{l}\text { CDA5-RC } \\
\text { partial }\end{array}$ & $\begin{array}{l}\text { CDA5-RA } \\
\text { partial }\end{array}$ & CDA5-RE & $\begin{array}{l}\text { CDA5-RB } \\
\text { partial }\end{array}$ \\
\hline $\begin{array}{l}\text { Egg Citrus macrophylla CLas- } \\
\text { Whole body }\end{array}$ & 56.64 & 415.8 & 4.89 & 22.79 & 423.69 & 16.46 & 0 & 0 & 6.95 & 9.07 \\
\hline $\begin{array}{l}\text { Nymph Citrus medica CLas+ } \\
\text { Low infection Whole body }\end{array}$ & 67.1 & 576.33 & 111.58 & 271.48 & 571.7 & 50.9 & 16.28 & 0 & 108.52 & 2.93 \\
\hline $\begin{array}{l}\text { Nymph Citrus sinensis } C \mathrm{Las}^{+} \\
\text {High infection Whole body }\end{array}$ & 71.33 & 513.82 & 100.65 & 247.03 & 374.09 & 78.16 & 3.45 & 0 & 145.78 & 3.22 \\
\hline $\begin{array}{c}\text { Nymph } C \text {. sinensis CLas- } \\
\text { Whole body }\end{array}$ & 78.22 & 533.18 & 36.7 & 242.93 & 453.44 & 31.57 & 0 & 0 & 77.51 & 1.43 \\
\hline $\begin{array}{l}\text { Nymph C. macrophylla CLas- } \\
\text { Whole body }\end{array}$ & 63.26 & 398.54 & 5.1 & 15.5 & 564.13 & 66.92 & 0 & 0 & 74.46 & 9.88 \\
\hline $\begin{array}{c}\text { Nymph Citrus spp. CLas- } \\
\text { Whole body }\end{array}$ & 118.31 & 171.29 & 2.5 & 11.24 & 822.56 & 15.1 & 3.36 & 37.94 & 41.04 & 7.32 \\
\hline $\begin{array}{l}\text { Nymph Citrus spp. CLas+ } \\
\text { Whole body }\end{array}$ & 58.83 & 343.75 & 0.36 & 8.66 & 260.62 & 13.61 & 0 & 33.96 & 6.56 & 8.04 \\
\hline Adult $C$. medica $C$ Las- Gut & 0.24 & 0.02 & 0 & 0.12 & 0.27 & 0 & 0 & 0.07 & 0.3 & 0 \\
\hline Adult C. medica $C$ Las + Gut & 0.59 & 0.09 & 0.01 & 0.32 & 0.54 & 0.02 & 0 & 0.05 & 0.4 & 0 \\
\hline $\begin{array}{l}\text { Adult } C \text {. medica } C \text { Las }+ \text { High } \\
\text { infection Whole body }\end{array}$ & 40.95 & 78.19 & 42.05 & 40.72 & 77.88 & 15 & 0 & 0 & 22.22 & 0.12 \\
\hline $\begin{array}{l}\text { Adult } C \text {. medica } C \text { Las+ Low } \\
\text { infection Whole body }\end{array}$ & 35.92 & 94.71 & 97.78 & 9.81 & 99.09 & 9.59 & 0 & 0 & 27.89 & 0.07 \\
\hline $\begin{array}{l}\text { Adult } C \text {. medica } C \text { Las- Whole } \\
\text { body }\end{array}$ & 46.28 & 171.94 & 125.69 & 78.25 & 193.02 & 29.12 & 4.81 & 0 & 48.75 & 0.2 \\
\hline $\begin{array}{l}\text { Adult C. macrophylla CLas- } \\
\text { Whole body }\end{array}$ & 5.48 & 16.96 & 0 & 0.53 & 15.86 & 2.51 & 0 & 0 & 3.67 & 0 \\
\hline $\begin{array}{l}\text { Adult Citrus spp. CLas- Whole } \\
\text { body }\end{array}$ & 14.83 & 0 & 0 & 1.41 & 66.18 & 2.36 & 0 & 0.39 & 1.78 & 0 \\
\hline $\begin{array}{l}\text { Adult Citrus spp. CLas+ Whole } \\
\text { body }\end{array}$ & 8.49 & 9.2 & 0 & 3.78 & 9.23 & 0.66 & 1.12 & 1.98 & 2.62 & 0 \\
\hline Adult Citrus spp. CLas- midgut & 0.73 & 0 & 0.03 & 0.5 & 3.13 & 0 & 0 & 0 & 0.17 & 0 \\
\hline Adult Citrus spp. CLas+ midgut & 3.36 & 5.45 & 0.02 & 0.07 & 11.56 & 0.13 & 0.01 & 0.12 & 1.02 & 0 \\
\hline $\begin{array}{l}\text { Adult Citrus reticulata CLas- } \\
\text { Female abdomen }\end{array}$ & 7.34 & 6.88 & 0 & 0.48 & 8.36 & 0.57 & 0 & 0 & 1.09 & 0 \\
\hline $\begin{array}{c}\text { Adult } C \text {. reticulata } C \text { Las- } \\
\text { Female antennae }\end{array}$ & 9.65 & 31.14 & 0.14 & 0.75 & 30.31 & 1.7 & 0 & 0 & 2.81 & 0 \\
\hline $\begin{array}{l}\text { Adult } C \text {. reticulata CLas- } \\
\text { Female head }\end{array}$ & 13.95 & 12.73 & 0.54 & 0.55 & 20.44 & 0 & 0 & 0.76 & 2.96 & 0 \\
\hline $\begin{array}{c}\text { Adult C. reticulata CLas- } \\
\text { Female leg }\end{array}$ & 17.46 & 4.67 & 0.01 & 6.6 & 12.63 & 0 & 0 & 1.16 & 1.7 & 0.48 \\
\hline $\begin{array}{l}\text { Adult C. reticulata CLas- } \\
\text { Female terminal abdomen }\end{array}$ & 14.73 & 36.64 & 0.08 & 1.27 & 16.78 & 0.53 & 0.08 & 0 & 3.73 & 0.76 \\
\hline $\begin{array}{c}\text { Adult } C \text {. reticulata } C \text { Las- } \\
\text { Female thorax }\end{array}$ & 15.3 & 7.91 & 0.04 & 0.03 & 15.29 & 2.53 & 0 & 0.2 & 0.97 & 0 \\
\hline $\begin{array}{l}\text { Adult } C \text {. reticulata } C \text { Las- Male } \\
\text { abdomen }\end{array}$ & 8.81 & 2.95 & 0 & 2.95 & 15.63 & 0.79 & 0 & 0.93 & 0.73 & 0 \\
\hline $\begin{array}{l}\text { Adult } C \text {. reticulata } C \text { Las- Male } \\
\text { antennae }\end{array}$ & 11.9 & 36.52 & 0.79 & 2.06 & 30.78 & 2.14 & 0 & 0 & 4.56 & 0.13 \\
\hline $\begin{array}{l}\text { Adult } C \text {. reticulata CLas- Male } \\
\text { head }\end{array}$ & 10.15 & 17.32 & 0.45 & 0.43 & 28.15 & 1.21 & 0 & 0 & 2.4 & 1.12 \\
\hline $\begin{array}{l}\text { Adult } C \text {. reticulata CLas- Male } \\
\text { leg }\end{array}$ & 17.7 & 4.13 & 0.02 & 6.26 & 13.4 & 0.88 & 0 & 0.09 & 2.19 & 0.41 \\
\hline $\begin{array}{l}\text { Adult } C \text {. reticulata } C \text { Las- Male } \\
\text { terminal abdomen }\end{array}$ & 17.41 & 20.3 & 1.29 & 0.75 & 25.87 & 1.9 & 0 & 0 & 5.19 & 0.16 \\
\hline $\begin{array}{l}\text { Adult } C \text {. reticulata } C \text { Las- Male } \\
\text { thorax }\end{array}$ & 16.31 & 8.55 & 0 & 1.62 & 17.42 & 1.65 & 0 & 0 & 4.11 & 0.32 \\
\hline $\begin{array}{c}\text { Adult } C \text {. reticulata CLas- } \\
\text { Female antennae [23] }\end{array}$ & 14.37 & 16.64 & 0.06 & 5.52 & 18.05 & 0.24 & 0.04 & 1.14 & 1.47 & 0 \\
\hline $\begin{array}{l}\text { Adult } C \text {. reticulata CLas- } \\
\text { Female terminal abdomen [23] }\end{array}$ & 5.83 & 11.74 & 0 & 2.95 & 4.61 & 0.58 & 0 & 0.14 & 0.48 & 0.12 \\
\hline
\end{tabular}




\begin{tabular}{|c|c|c|c|c|c|c|c|c|c|c|}
\hline Gene ID & $\begin{array}{l}\text { Dcitr04g03 } \\
\text { 590.1.1 }\end{array}$ & $\begin{array}{l}\text { Dcitr04g03 } \\
590.1 .2\end{array}$ & $\begin{array}{l}\text { Dcitr04g03 } \\
540.1 .1\end{array}$ & $\begin{array}{l}\text { Dcitr04g03 } \\
540.1 .2\end{array}$ & $\begin{array}{l}\text { Dcitr02g03 } \\
\text { 950.1.1 }\end{array}$ & $\begin{array}{l}\text { Dcitr01g12 } \\
\text { 310.1.1 }\end{array}$ & $\begin{array}{l}\text { Dcitr01g12 } \\
310.1 .2\end{array}$ & $\begin{array}{l}\text { Dcitr01g12 } \\
310.1 .3\end{array}$ & $\begin{array}{l}\text { Dcitr01g12 } \\
\text { 310.1.4 }\end{array}$ & $\begin{array}{l}\text { Dcitr01g12 } \\
310.1 .5\end{array}$ \\
\hline Gene/Transcript Name & CDA1-RB & CDA1-RA & CDA2-RB & CDA2-RA & CDA4 & CDA5-RD & $\begin{array}{l}\text { CDA5-RC } \\
\text { partial }\end{array}$ & $\begin{array}{l}\text { CDA5-RA } \\
\text { partial }\end{array}$ & CDA5-RE & $\begin{array}{l}\text { CDA5-RB } \\
\text { partial }\end{array}$ \\
\hline $\begin{array}{c}\text { Adult } C \text {. reticulata CLas- Male } \\
\text { antennae [23] }\end{array}$ & 8.22 & 26.46 & 0.91 & 0.73 & 17.65 & 0.3 & 0 & 0 & 5.71 & 2.59 \\
\hline $\begin{array}{l}\text { Adult } C \text {. reticulata CLas- Male } \\
\text { terminal abdomen [23] }\end{array}$ & 11.37 & 6.91 & 0 & 3.67 & 12.05 & 0.97 & 0.09 & 0 & 2.68 & 0.1 \\
\hline
\end{tabular}

\begin{tabular}{|c|c|c|c|c|c|c|}
\hline Species & Group I & Group II & Group III & Group IV & Group V & Total \\
\hline D. melanogaster & $2^{*}$ & 1 & 1 & $1^{*}$ & 1 & 6 \\
\hline A. gambiae & $2^{*}$ & 1 & 1 & 1 & 0 & 5 \\
\hline T. castaneum & $2^{*}$ & 1 & 1 & $1^{*}$ & 4 & 9 \\
\hline B. mori & $2^{*}$ & 1 & 1 & 1 & 3 & 8 \\
\hline A. mellifera & $2^{*}$ & 1 & 1 & $1^{*}$ & 0 & 5 \\
\hline N. vitripennis & 2 & 1 & 1 & 1 & 0 & 5 \\
\hline R. prolixus & 2 & 0 & 1 & 1 & 0 & 4 \\
\hline A. pisum & 2 & 0 & 1 & 1 & 0 & 4 \\
\hline$N$. lugens & 2 & 0 & 1 & 1 & 0 & 4 \\
\hline D. citri & $2^{*}$ & 0 & 1 & $1^{*}$ & 0 & 4 \\
\hline \multicolumn{7}{|c|}{$\begin{array}{l}\text { D. citri gene numbers were determined based on annotation of the } D \text {. citri genome v3. All other ortholog number } \\
\text { were obtained from published sources }[5,6,24-32] \text {. An asterisk }(*) \text { indicates that isoforms have been found for at } \\
\text { least one member of the group in that organism. }\end{array}$} \\
\hline
\end{tabular}

\begin{tabular}{|c|c|c|c|c|c|c|}
\hline \multirow[t]{2}{*}{ Gene/ Isoform } & \multirow[t]{2}{*}{ OGSv3 ID } & \multirow{2}{*}{$\begin{array}{l}\text { Gene model } \\
\text { Complete }\end{array}$} & \multicolumn{4}{|c|}{ Evidence supporting annotation } \\
\hline & & & MCOT & Iso-Seq & RNA-Seq & Ortholog \\
\hline \multirow[t]{2}{*}{ CDA1 (Group I) } & Dcitr04g03590.1.1 & $\mathrm{X}$ & МСОТ00151.2.СО & $\mathrm{X}$ & $\mathrm{X}$ & X \\
\hline & Dcitr04g03590.1.2 & & МСОТ00151.1.CO & & & \\
\hline \multirow[t]{2}{*}{ CDA2 (Group I) } & Dcitr04g03540.1.1 & $\mathrm{X}$ & & $\mathrm{X}$ & $\mathrm{X}$ & $\mathrm{X}$ \\
\hline & Dcitr04g03540.1.2 & & & & & \\
\hline CDA3 (Group III) & Dcitr02g03950.1.1 & $\mathrm{X}$ & МСОТ04789.0.СТ & $\mathrm{x}$ & & $\mathrm{X}$ \\
\hline \multirow[t]{5}{*}{ CDA5 (Group IV) } & Dcitr01g12310.1.1 & & МСОТ14896.0.СТ & $\mathrm{X}$ & $\mathrm{X}$ & $\mathrm{X}$ \\
\hline & Dcitr01g12310.1.2 & & МСОТ06229.1.СO & & & \\
\hline & Dcitr01g12310.1.3 & & МСОТ06229.3.CO & & & \\
\hline & Dcitr01g12310.1.4 & & МСОТ19482.0.СТ & & & \\
\hline & Dcitr01g12310.1.5 & & MCOT06229.2.CO & & & \\
\hline \multicolumn{7}{|c|}{$\begin{array}{l}\text { MCOT: MAKER (MAKER, RRID:SCR_005309), Cufflinks (Cufflinks, RRID:SCR_014597), Oases (Oases, } \\
\text { RRID:SCR_011896), Trinity (Trinity, RRID:SCR_013048) pipeline. Each manually annotated gene has been assigned } \\
\text { an OGSv3.0 gene identifier. Genes not marked as complete were only able to be annotated as partial gene models. } \\
\text { Evidence types used for manual annotation of each gene are indicated. More information on these evidence } \\
\text { sources is available in [16]. }\end{array}$} \\
\hline
\end{tabular}

curated CDA3 model from genome v3 has additional 3' sequence than the previously reported model (GenBank accession number XM_008481889.1), which was based on genome v1.1 [33,34]. The resulting predicted protein is almost 50 amino acids longer, with additional conserved sequence at the $\mathrm{C}$-terminus.

Yu et al. [33] reported that RNAi knockdown of $C D A 3$ had no effect on molting or wing development. Instead, their results implicated $C D A 3$ in the $D$. citri bacterial immune 

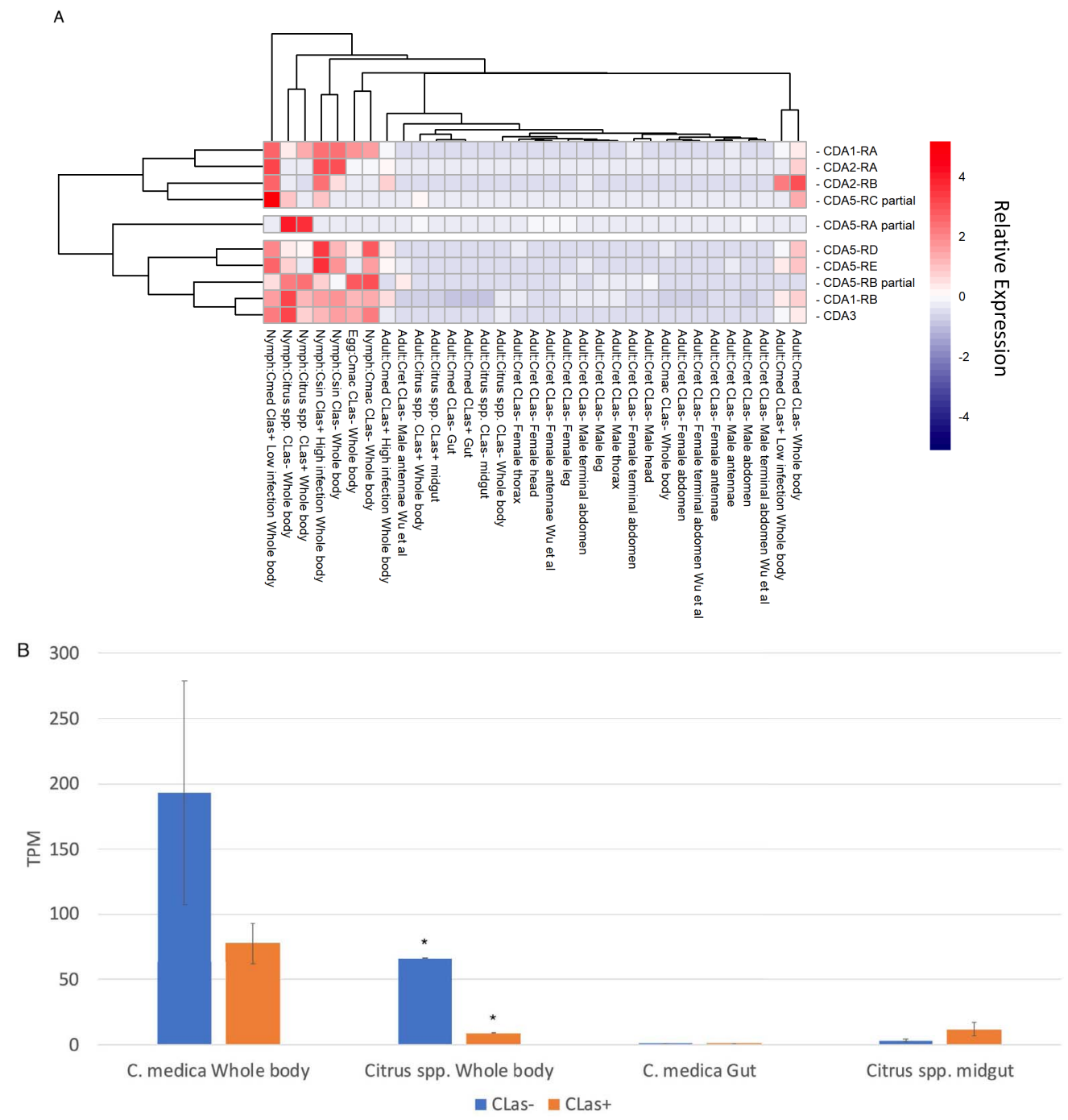

Figure 2. Expression of D. citri chitin deacetylase genes. (A) Heatmap displaying relative expression levels of all annotated chitin deacetylase genes in RNA-seq datasets from various life stages, tissues and CLas infection states. Expression levels were obtained from CGEN [20] and are reported in Table 2. The heatmap is scaled by row. (B) Expression levels (transcripts per million, TPM) of CDA3 (Dcitr02g03950.1.1) in tissues from CLas+ and CLaspsyllids fed on two different types of citrus plants. Standard error bars are shown for all expression values except those marked with an asterisk (*), which had only one replicate. Expression levels were obtained from CGEN [20].

response. Recombinant CDA3 showed antibacterial activity against gram-positive bacteria, but had no effect on gram-negative bacteria. Moreover, injection of either Escherichia coli (gram-negative) or Staphylococcus aureus (gram-positive) bacteria into D. citri increased $C D A 3$ expression in the midgut and decreased its expression in the fat body, although the timeline of these effects is not certain. To determine whether infection by CLas, a gram-negative bacterium, might also affect $C D A 3$ expression, we used CGEN [20] to compare expression of $D$. citri CDA3 in RNA-seq datasets from CLas+ and CLas- guts [35], midguts [36] and whole bodies ([37] and NCBI BioProject PRJNA609978). CDA3 expression was lower in CLas+ versus CLas- whole body tissue in data from two different RNA-Seq experiments (Figure 2B). Expression of CDA3 in midgut and gut tissues was low in all 


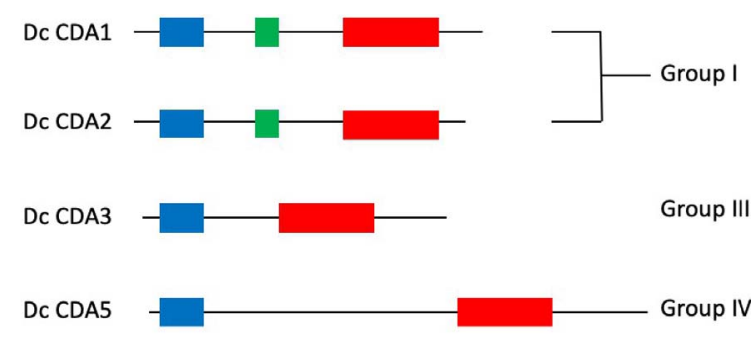

Deacetylase Catalytic Domain

Chitin Binding Domain

Low-Density Lipoprotein Receptor Class A Domain

Linker Region

Figure 3. Chitin deacetylase domain organization in $D$. citri. Chitin deacetylases are categorized by group based on phylogenetic analysis, sequence similarity, and domain organization. $D$. citri domain analysis was performed using InterPro [17]. CDA5 is represented by the protein encoded by the de novo-assembled transcript MCOT06229.1.CO [34] because a small portion of the CDA5 gene is missing from the v3 genome assembly.

samples but there was a slight increase in CLas+ versus CLas- midgut expression (Figure 2B). While the significance of these expression differences is not clear, they may warrant further investigation.

\section{Group IV chitin deacetylases}

Most insects examined to date have one group IV CDA, typically called CDA5 (CDA4 in $N$. lugens) (Table 3). CDA5 has been shown to have multiple isoforms in Tribolium and Drosophila [5]. Consistent with these observations, we identified and annotated five different isoforms of CDA5 in D. citri (Table 4). Unfortunately, the annotated models are missing a small amount of 3 ' sequence due to genome assembly issues. However, we identified a de-novo assembled transcript (MCOT06229.1.CO) that appears to encode the full-length protein (Figure 3). The missing genome sequence does not affect the conserved functional domains of CDA5. Four of the five transcript isoforms encode proteins containing both an N-terminal ChBD and a C-terminal catalytic domain, as seen in other insect CDA5 orthologs. The remaining isoform (CDA5-RB) differs at the 5' end and apparently lacks a ChBD-encoding region.

\section{Other chitin deacetylase groups}

We did not find any group II or group V CDAs in the $D$. citri v3 genome (Figure 4). To our knowledge CDAs from these groups have not been found in any hemipteran insects examined to date [6], so their absence in $D$. citri was expected.

\section{RE-USE POTENTIAL}

This manual curation was carried out as a part of the Diaphorina citri community annotation project [38] with a goal to annotate gene families related to immune response, metabolism and other major functions [39-42]. Our manual annotations will be useful for researchers studying the chitin deacetylase genes in the future. We annotated multiple isoforms for three of the four genes, which will inform the design of experiments to 


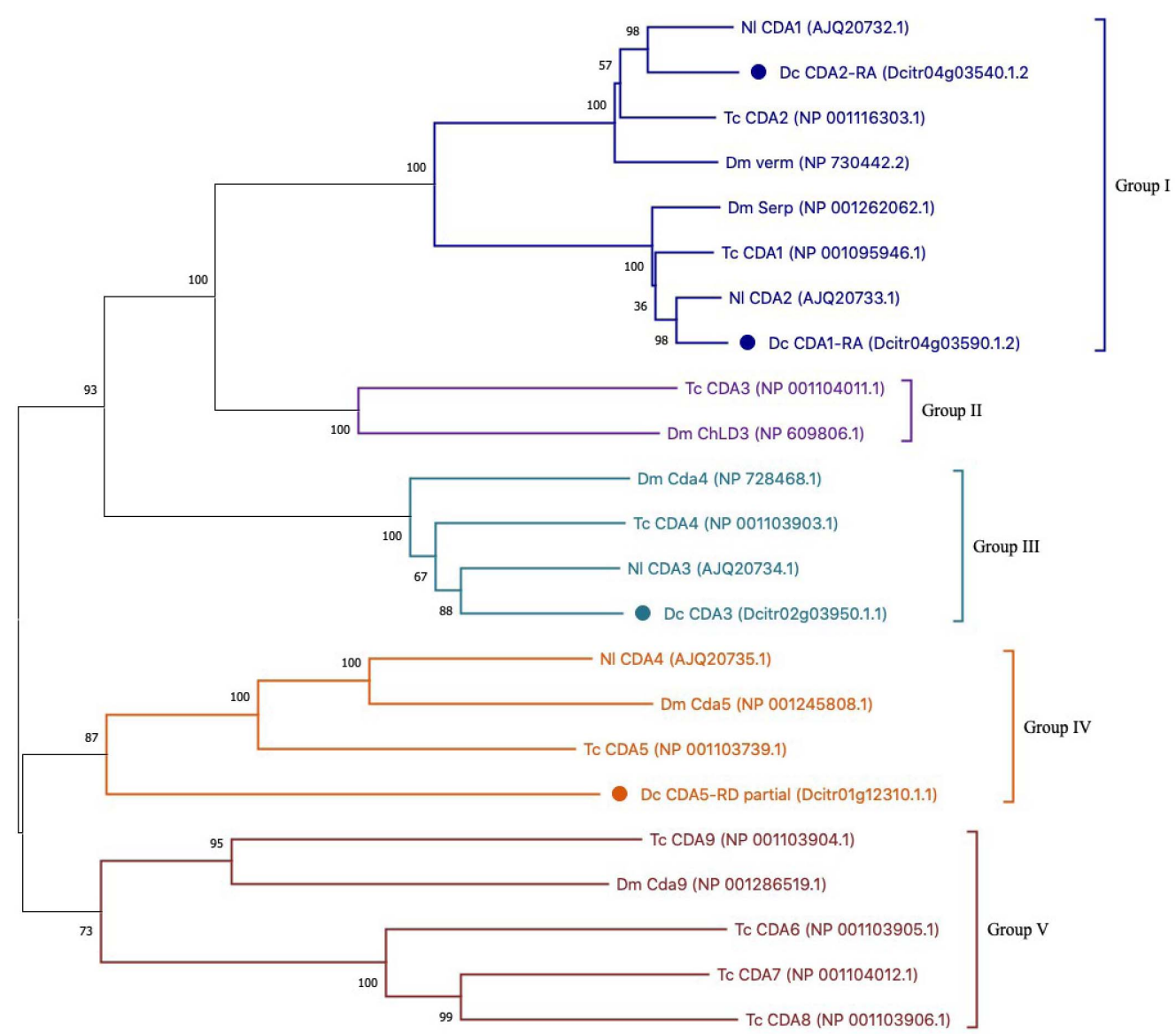

0.10

Figure 4. Phylogenetic tree of chitin deacetylase family members. ClustalW was used to perform the multiple sequence alignment. The tree was constructed with MEGA X software [19] using neighbor-joining analysis with 100 bootstrap replications. Drosophila melanogaster (Dm), Tribolium castaneum (Tc), Nilaparvata lugens (Nl), and Diaphorina citri (Dc). D. citri protein branches are marked with a circle. Colors delineate established chitin deacetylase groups.

determine the expression pattern and function of specific isoforms. Our annotations will be incorporated into an updated official gene set and will be publicly available for comparative expression profiling on the CGEN [20].

\section{DATA AVAILABILITY}

The Diaphorina citri genome assembly, gene sets, and transcriptome data are accessible via the Citrus Greening website [20]. All accessions for genes used for phylogenetic analysis are provided within this report, and all additional data is available via the GigaScience GigaDB repository [43]. 


\section{EDITOR'S NOTE}

This article is one of a series of Data Releases crediting the outputs of a student-focused and community-driven manual annotation project curating gene models and if required, correcting assembly anomalies, for the Diaphorina citri genome project [13].

\section{DECLARATIONS}

\section{LIST OF ABBREVIATIONS}

CDA: chitin deacetylase; CGEN: Citrus Greening Expression Network; ChBD: chitin binding domain; CLas: Candidatus Liberibacter asiaticus ; NCBI: National Center for Biotechnology Information; RNAi: RNA interference; TPM: transcripts per million.

\section{ETHICAL APPROVAL}

Not applicable.

\section{CONSENT FOR PUBLICATION}

Not applicable.

\section{COMPETING INTERESTS}

The authors declare that they have no competing interests.

\section{FUNDING}

This research was funded by USDA-NIFA grant 2015-70016-23028, HSI 1300394 , 2020-70029-33199 and an Institutional Development Award (IDeA) from the National Institute of General Medical Sciences of the National Institutes of Health under grant number P20GM103418.

\section{AUTHORS' CONTRIBUTIONS}

WBH, SJB, TD and LAM conceptualized the study; TD, SS, TDS and SJB supervised the study; SJB, TD, SS, and LAM contributed to project administration; SM, TDS, and BT conducted investigation; PH, MF-G, and SS contributed to software development; SS, TDS, PH, and MF-G developed methodology; SJB, TD, WBH, and LAM acquired funding; SM and TDS prepared and wrote the original draft; SS, WBH and SJB reviewed and edited the draft.

\section{ACKNOWLEDGEMENTS}

We thank Dr. Josh Benoit for assistance with data visualization.

\section{REFERENCES}

1 Kaczmarek MB, Struszczyk-Swita K, Li X, Szczęsna-Antczak M, Daroch M, Enzymatic modifications of chitin, chitosan, and chitooligosaccharides. Front. Bioeng. Biotechnol., 2019; 7: 243.

2 Guo W, Li G, Pang Y, Wang P, A novel chitin-binding protein identified from the peritrophic membrane of the cabbage looper, Trichoplusia ni. Insect Biochem. Mol. Biol., 2005; 35: 1224-1234.

3 Luschnig S, Bätz T, Armbruster K, Krasnow MA, serpentine and vermiform encode matrix proteins with chitin binding and deacetylation domains that limit tracheal tube length in Drosophila. Curr. Biol., 2006; 16: 186-194.

4 Wang S et al. Septate-junction-dependent luminal deposition of chitin deacetylases restricts tube elongation in the Drosophila trachea. Curr. Biol., 2006; 16: 180-185.

5 Dixit $\mathbf{R}$ et al. Domain organization and phylogenetic analysis of proteins from the chitin deacetylase gene family of Tribolium castaneum and three other species of insects. Insect Biochem. Mol. Biol., 2008; 38: 440-451. 
6 Xi Y, Pan PL, Ye YX, Yu B, Zhang CX, Chitin deacetylase family genes in the brown planthopper, Nilaparvata lugens (Hemiptera: Delphacidae). Insect Mol. Biol., 2014; 23: 695-705.

7 Noh MY, Muthukrishnan S, Kramer KJ, Arakane Y, Group I chitin deacetylases are essential for higher order organization of chitin fibers in beetle cuticle. J. Biol. Chem., 2018; 293: 6985-6995.

8 Liu X, Cooper AMW, Yu Z, Silver K, Zhang J, Zhu KY, Progress and prospects of arthropod chitin pathways and structures as targets for pest management. Pestic. Biochem. Physiol., 2019; 161: $33-46$.

9 Quan G et al. Characterization of a spruce budworm chitin deacetylase gene: Stage- and tissue-specific expression, and inhibition using RNA interference. Insect Biochem. Mol. Biol., 2013; 43: 683-691.

10 Yan $\mathrm{X}$ et al. Identification and characterization of chitin deacetylase2 from the American white moth, Hyphantria cunea (Drury). Gene, 2018; 670: 98-105.

11 Yang W-J et al. Functional characterization of chitin deacetylase 1 gene disrupting larval-pupal transition in the drugstore beetle using RNA interference. Comp. Biochem. Physiol. B Biochem. Mol. Biol., 2018; 219-220: 10-16.

12 Wu J-J, Chen Z-C, Wang Y-W, Fu K-Y, Guo W-C, Li G-Q, Silencing chitin deacetylase 2 impairs larval-pupal and pupal-adult molts in Leptinotarsa decemlineata. Insect Mol. Biol., 2019; 28: 52-64.

13 Hosmani P et al. Chromosomal length reference assembly for Diaphorina citri using single-molecule sequencing and Hi-C proximity ligation with manually curated genes in developmental, structural and immune pathways. bioRxiv. 2019; 869685. https://doi.org/doi:10.1101/869685.

14 National Center for Biotechnology Information (NCBI). Ref-Seq Non-redundant Protein Database. Bethesda, MD: NCBI. https://www.ncbi.nlm.nih.gov/refseq/about/nonredundantproteins/. Accessed 04 Nov 2020.

15 Dunn NA et al. Apollo: Democratizing genome annotation. PLOS Comput. Biol., 2019; 15: e1006790.

16 Shippy TD et al. Annotating genes in Diaphorina citri genome version 3. protocols.io. 2020; http://doi.org/10.17504/protocols.io.bniimcce.

17 European Molecular Biology Laboratory-European Bioinformatics Institute (EMBL-EBI). InterPro. Hinxton: EMBL-EBI. https://www.ebi.ac.uk/interpro/. Accessed 04 Nov 2020.

18 Larkin MA et al. Clustal W and Clustal X version 2.0. Bioinformatics, 2007; 23: 2947-2948.

19 Kumar S, Stecher G, Li M, Knyaz C, Tamura K, MEGA X: Molecular evolutionary genetics analysis across computing platforms. Mol. Biol. Evol., 2018; 35: 1547-1549.

20 Flores-Gonzalez $\mathbf{M}$ et al. Citrusgreening.org: An open access and integrated systems biology portal for the Huanglongbing (HLB) disease complex. bioRxiv. 2019; 868364. https://doi.org/10.1101/868364.

21 R Core Team. R: A language and environment for statistical computing. Vienna: R Foundation for Statistical Computing 2020; https://www.R-project.org/. Accessed December 172020.

22 Kolde R, pheatmap: Pretty Heatmaps (Version 1.0.12). 2020; https://cran.r-project.org/package=pheatmap.

23 Wu Z, Zhang H, Bin S, Chen L, Han Q, Lin J, Antennal and abdominal transcriptomes reveal chemosensory genes in the Asian Citrus Psyllid, Diaphorina citri. PLoS One, 2016; 11: e0159372.

24 Adams MD et al. The genome sequence of Drosophila melanogaster. Science, 2000; 2185-2195.

25 Holt RA et al. The genome sequence of the malaria mosquito Anopheles gambiae. Science, 2002; 298: 129-149.

26 Richards S et al. Tribolium Genome Sequencing Consortium. The genome of the model beetle and pest Tribolium castaneum. Nature, 2008; 452: 949-955.

27 Kawamoto $\mathbf{M}$ et al. High-quality genome assembly of the silkworm, Bombyx mori. Insect Biochem. Mol. Biol., 2019; 107: 53-62.

28 Elsik CG et al. Finding the missing honey bee genes: lessons learned from a genome upgrade. BMC Genomics, 2014; 15: 86. doi:10.1186/1471-2164-15-86.

29 Werren JH et al. Functional and evolutionary insights from the genomes of three parasitoid Nasonia species. Science, 2010; 327: 343-348, doi:10.1126/science.1178028.

30 Mesquita RD et al. Genome of Rhodnius prolixus, an insect vector of Chagas disease, reveals unique adaptations to hematophagy and parasite infection. Proc. Natl Acad. Sci. USA, 2015; 112: 14936-14941.

31 International Aphid Genomics Consortium. Genome sequence of the pea aphid Acyrthosiphon pisum. PLoS Biol., 2010; 8: e1000313. doi:10.1371/journal.pbio.1000313. 
32 Ma W et al. Chromosomal-level genomes of three rice planthoppers provide new insights into sex chromosome evolution. Mol. Ecol. Resour., 2020; 21: 226-237, doi:10.1111/1755-0998.13242.

33 Yu HZ et al. Immune functional analysis of Chitin Deacetylase 3 from the Asian citrus psyllid Diaphorina citri. Int. J. Mol. Sci., 2020; 21: 64.

34 Saha S et al. Improved annotation of the insect vector of citrus greening disease: biocuration by a diverse genomics community. Database 2017. 2017; bax032. https://dx.doi.org/10.1093/database/bax032.

35 Kruse A et al. Combining 'omics and microscopy to visualize interactions between the Asian citrus psyllid vector and the Huanglongbing pathogen Candidatus Liberibacter asiaticus in the insect gut. PLoS One, 2017; 12: e0179531.

36 Yu HZ et al. Transcriptome analyses of Diaphorina citri midgut responses to Candidatus Liberibacter asiaticus infection. Insects, 2020; 11: 171.

37 Vyas $\mathbf{M}$ et al. Asian citrus psyllid expression profiles suggest Candidatus liberibacter asiaticus-mediated alteration of adult nutrition and metabolism, and of nymphal development and immunity. PLoS One, 2015; 10: e0130328.

38 Citrus Greening Solutions. Annotation of psyllid genome. 2018;

https://citrusgreening.org/annotation/index. Accessed 18 December 2020.

39 Vosburg $\mathbf{C}$ et al. Utilizing a chromosomal-length genome assembly to annotate the Wnt signaling pathway in the Asian citrus psyllid, Diaphorina citri. Gigabyte, 2021; 1: https://doi.org/10.46471/gigabyte.21.

40 Massimino C et al. Annotation of yellow genes in Diaphorina citri, the vector for Huanglongbing disease. Gigabyte, 2021; 1: https://doi.org/10.46471/gigabyte.20.

41 Miller S et al. Annotation of chitin biosynthesis genes in Diaphorina citri, the Asian citrus psyllid. Gigabyte, 2021; 1: https://doi.org/10.46471/gigabyte.23.

42 Miller S et al. Segmentation pathway genes in the Asian citrus psyllid, Diaphorina citri. bioRxiv. 2020; https://doi.org/10.1101/2020.12.24.424320.

43 Miller S, Shippy TD, Tamayo B, Hosmani PS, Flores-Gonzalez M, Mueller LA, Hunter WB, Brown SJ, D'Elia T, Saha S, Supporting data for "In silico characterization of chitin deacetylase genes in the Diaphorina citri genome”. GigaScience Database. 2021; http://dx.doi.org/10.5524/100901. 